\title{
Wave Scattering by One and Many Thin Material Strips: Singular Integral Equations, Meshless Nystrom Discretization, and Periodicity Caused Resonances
}

\author{
Olga V. Shapoval ${ }^{1}$, Ilya O. Sukharevsky ${ }^{2}$, Ayhan Altintas ${ }^{2}$, Ronan Sauleau ${ }^{3}$, Alexander I. Nosich ${ }^{1}$ \\ ${ }^{1}$ Laboratory of Micro and Nano Optics, Institute of Radio-Physics and Electronics NASU, Ukraine, olga.v.shapoval@gmail.com \\ ${ }^{2}$ Department of Electrical and Electronics Engineering, Bilkent University, Ankara, Turkey, i.sukharevsky@ gmail.com \\ ${ }^{3}$ I nstitut d'Electronique et Telecommunications de Rennes, Universite de Rennes 1, Rennes, France
}

\begin{abstract}
We consider the medial-line singular-integral equation technique for the analysis of the scattering by multiple thin material strips. Their discretization is performed using the Nystrom-type scheme that guarantees convergence. Numerical study of the scattering by periodic arrays of a few hundred or more strips reveals specific high- $Q$ resonances caused by the periodicity.
\end{abstract}

Index Terms - thin strip, scattering, integral equation.

\section{INTRODUCTION}

Dielectric and metal thinner-than-wavelength strips are increasingly attractive as easily manufactured components of both microwave and optical antenna circuits. In particular, metal nanostrips illuminated by a transversely-polarized light are known to display intensive localized surface-plasmon resonances in the visible and far-infrared ranges, used to design sensors of the host-medium refractive index. The typical dimensions of metal nanostrips are the width from 100 to $1000 \mathrm{~nm}$ and the thickness from 5 to $50 \mathrm{~nm}$ that is 8 to 180 times smaller than the wavelength in the whole visible band (400 to $900 \mathrm{~nm}$ ).

In parallel to rough FDTD codes, the volume and boundary integral equations (IEs), where the integration domain is the area of the strip cross-section and its closed contour, respectively, are among the most advanced computational methods used in the optical modeling of nanostrips. Boundary IEs are considered more economic however typical number of unknowns is in thousands and still many forms of them possess spurious eigenvalues (real-valued false eigenfrequencies). To avoid this, one should use so-called Muller's boundary IE with smooth and integrable kernels. Still the contour shape and its smoothness greatly affect the rate of convergence. All mentioned becomes even more important for multiple metal strips.

Practical suggestion is that, in the case of a thin strip, the analysis can be simplified by neglecting the internal field. This enables reduction of the integration contour to the strip median line and use of the generalized boundary conditions (GBC),

$$
\begin{aligned}
(1 / 2)\left[\vec{E}_{T}^{+}(x, 0)+\vec{E}_{T}^{-}(x, 0)\right] & =R Z_{0} \vec{n} \times\left[\vec{H}_{T}^{+}(x, 0)-\vec{H}_{T}^{-}(x, 0)\right], \\
(1 / 2)\left[\vec{H}_{T}^{+}(x, 0)+\vec{H}_{T}^{-}(x, 0)\right] & =-Q Z_{0}^{-1} \vec{n} \times\left[\vec{E}_{T}^{+}(x, 0)-\vec{E}_{T}^{-}(x, 0)\right],
\end{aligned}
$$

$$
L_{j}=\{(x, y): x \in[(j-1) p,(j-1) p+d], y=0\} \quad \vec{E}^{i n c}
$$

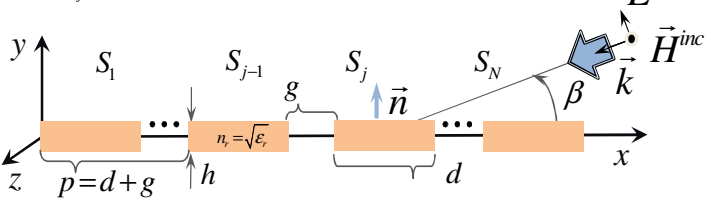

(a)

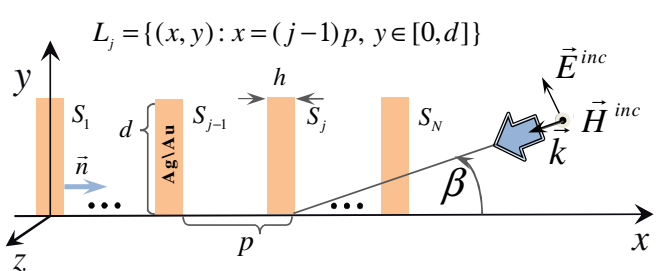

(b)

Fig. 1 Cross-sectional geometries of finite gratings made of $N$ thin material strips illuminated by a plane wave: flat grating (a) and comb grating (b).

This idea has been already exploited in the 1980s-1990s in relation to the shells and strips of conventional dielectrics [14]; since recently it has been applied also to the noble-metal nanostrips in the visible range [5-8]. Note that (1) has the meaning of Ohm's law, where R and Q are the relative electric and magnetic resistivities (see $[1-4,8]), Z_{0}$ is the free-space impedance, and $\vec{n}$ is the normal unit vector as shown in Fig. 1. Justification of (1) for dielectric and metal strips can be found in [8] where the GBC-based numerical data have been compared to the data obtained using the Muller boundary IE. 


\section{SINGULAR INTEGRAL EQUATIONS}

\section{A. Singular Integral Equations}

To satisfy Helmholtz equation and radiation condition, we seek the scattered field as a sum of the single-layer and doublelayer potentials, where unknown functions are effective magnetic and electric currents at $L_{j}, j=1, \ldots, N$ :

$$
H_{z}^{s c}(\vec{r})=k \sum_{j=1}^{N} \int_{L_{j}} v_{j}\left(\vec{r}^{\prime}\right) G\left(\vec{r}, \vec{r}^{\prime}\right) d \vec{r}^{\prime}+\sum_{j=1}^{N} \int_{L_{j}} w_{j}\left(\vec{r}^{\prime}\right) \frac{\partial G\left(\vec{r}, \vec{r}^{\prime}\right)}{\partial \vec{n}^{\prime}} d \vec{r}^{\prime}(2)
$$

Using GBC, we obtain two sets of coupled SIEs of the second kind with logarithmic and hyper-type singularities,

$$
\begin{gathered}
i Q v_{s}\left(\vec{r}_{0}\right)+\sum_{j=1}^{N} \int_{L_{j}} w_{j}(\vec{r}) \frac{\partial G\left(\vec{r}, \vec{r}_{0}\right)}{\partial \vec{n}(\vec{r})} d \vec{r}+ \\
k \sum_{j=1}^{N} \int_{L_{j}} v_{j}(\vec{r}) G\left(\vec{r}, \vec{r}_{0}\right) d \vec{r}=-u_{0}\left(\vec{r}_{0}\right), \\
i k R w_{s}\left(\vec{r}_{0}\right)+k \sum_{j=1}^{N} \int_{L_{j}} v_{j}(\vec{r}) \frac{\partial G\left(\vec{r}, \vec{r}_{0}\right)}{\partial \vec{n}\left(\vec{r}_{0}\right)} d \vec{r}+ \\
\sum_{j=1}^{N} \int_{L_{j}} w_{j}(\vec{r}) \frac{\partial G\left(\vec{r}, \vec{r}_{0}\right)}{\partial \vec{n}\left(\vec{r}_{0}\right) \partial \vec{n}(\vec{r})} d \vec{r}=-\partial u_{0}\left(\vec{r}_{0}\right) / \partial \vec{n}\left(\vec{r}_{0}\right), \vec{r}_{0} \in L_{s}
\end{gathered}
$$

In the case of flat grating of Fig. 1 (a) these sets decouple.

\section{B. Nystrom-type Discretization}

Nystrom methods of discretization of various IEs are actively developed today $[3,9,10]$. We have recently built a novel technique of this type and applied it to the scattering by flat dielectric and metal strips characterized with GBC and SIE $[4,5,7,8]$. The associated quadrature formulas ensure rapid convergence (at least as $1 / M$, where $M$ is the order of interpolation polynomial used to approximate the current on each strip) of numerical solutions to the accurate ones. For instance, to achieve 4-digit accuracy in the analysis of a $2 \lambda$ wide silver strip, 20 equations are enough. Thanks to this fact, computing a 100 -element grating of $2 \lambda$-wide silver strips needs inverting a matrix of $M=2000$ only

\section{PERIODICITY CAUSED RESONANCES ON GRATINGS}

The scattered field in the far zone is

$$
\begin{gathered}
U_{s c}(\varphi) \sim(2 / i \pi k r)^{1 / 2} e^{i k r} \Phi(\varphi), r \rightarrow \infty, \\
\Phi(\varphi)=(i k / 4) \sum_{j=1}^{N} \int_{L_{j}}\left[v_{j}(x)-i \sin \varphi w_{j}(x)\right] e^{-i k \cos \varphi} d x
\end{gathered}
$$

for a flat grating of strips, and

$$
\begin{array}{r}
\Phi(\varphi)=(i k / 4) \sum_{j=1}^{N} \int_{L_{j}} v_{j}(y) e^{-i k\left(x_{j} \cos \varphi+y \sin \varphi\right)} d y- \\
(k / 4) \cos \varphi \int_{L_{j}} w_{j}(y) e^{-i k\left(x_{j} \cos \varphi+y \sin \varphi\right)} d y
\end{array}
$$

for a comb-like grating of $N$ strips. The total scattering crosssection (TSCS) is computed as

$$
\sigma_{s c}=(2 / \pi k) \int_{0}^{2 \pi}|\Phi(\varphi)|^{2} d \varphi
$$

and the absorption cross-section (ACS) is found by the integration of the power lost in the lossy scatterer [4,5].

To characterize the complex dielectric permittivity of silver we have used the experimental data of [11] smoothed with the aid of an Akima cubic spline interpolation

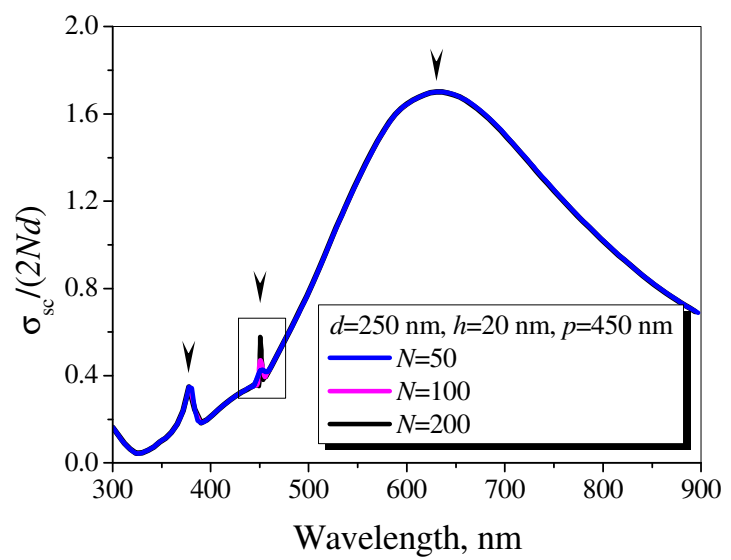

Fig. 2. Normalized TSCS versus the wavelength for the flat gratings of $N=$ $50,100,200$ strips of $d=250 \mathrm{~nm}, h=20 \mathrm{~nm}$ and $p=450 \mathrm{~nm}$. The Hpolarized plane wave is normally incident $(\beta=\pi / 2)$.

As mentioned, metal strip illuminated by the H-polarized light displays surface-plasmon resonances in the visible range. If several or many silver nanostrips are collected together into a flat grating, these resonances remain visible - see broad peak around $630 \mathrm{~nm}$ on the wavelength dependences of the normalized by $N$ values of TSCS in Fig. 2 .

However if a flat grating consists of dozens or hundreds of strips, new interesting effect appears induced by the periodic structuring. Computation of the wavelength dependences of both TSCS and ACS demonstrates the existence of the grating resonances also known as geometrical, lattice or collective resonances, near to but always red-shifted from the Rayleigh anomalies (see a sharper peak near $450 \mathrm{~nm}$ in Fig. 2). This is explained by the existence of specific poles of the field as a function of the wavelength and corresponding natural modes of a grating as a periodic open resonator.

In Fig. 3, we present two near-field patterns at the resonance wavelengths. They clearly show the different nature of these phenomena. The surface-plasmon resonance shows spots of high intensity just near each strip's boundary. In contrast, the grating resonance supports extended standingwave with bright spots both at and between the strips, stretching far from the plane of the grating. 


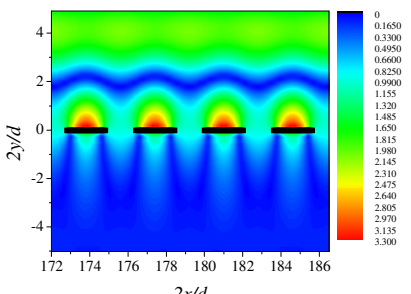

(a)

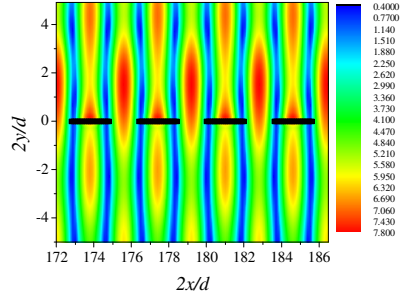

(b)
Fig. 3. In-resonance magnetic near-field patterns near four central strips of the $N=100$ strip grating: surface-plasmon resonance at $\lambda=632.3 \mathrm{~nm}$ (a) and grating resonance at $\lambda=$ $450.76 \mathrm{~nm}$ (b) for the same grating as in Fig. 2.

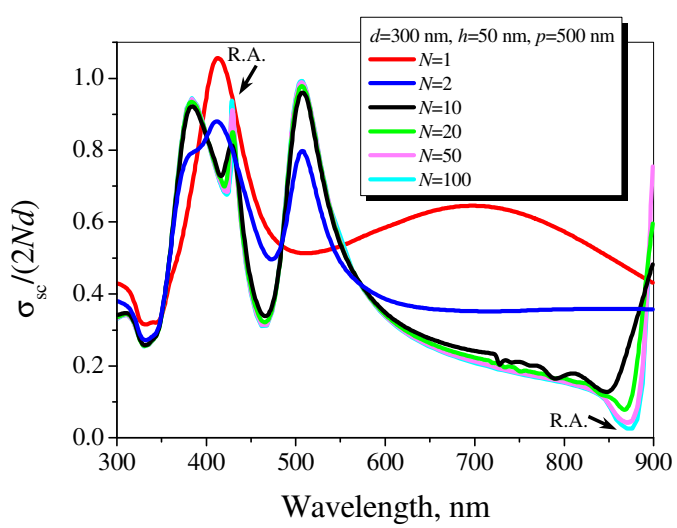

(a)

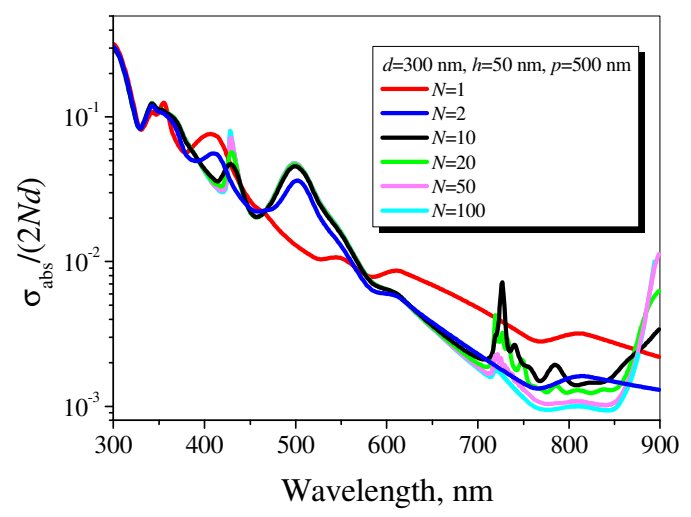

(b)

Fig. 4 Normalized TSCS (a) and ACS (b) as a function of the wavelength for $N$ silver strip grating of $d=300 \mathrm{~nm}, h=50 \mathrm{~nm}$ and $p=500 \mathrm{~nm}$ for the inclinely incident H-polarized plane wave $(\beta=\pi / 4)$. Rayleigh anomalies are at $\lambda=858.3$ $\mathrm{nm}(-1$-st) and $\lambda=428.7 \mathrm{~nm}$ (-2-nd).

A comb-like grating composed of silver nanostrips shows similar features in the optical scattering. The difference is in the existence of non-vanishing "grating domain" that entails associated family of natural modes called cavity modes, in addition to the surface plasmons and grating modes. The resonances on all these modes are seen in Fig. 4, and the field portraits at the resonance wavelengths are shown in Fig. 5.

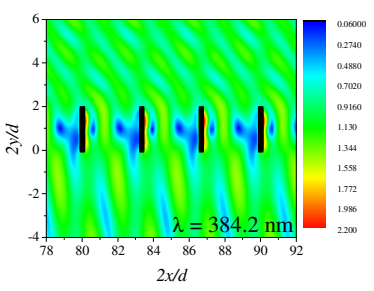

(a)

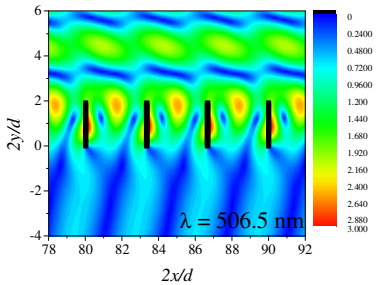

(c)

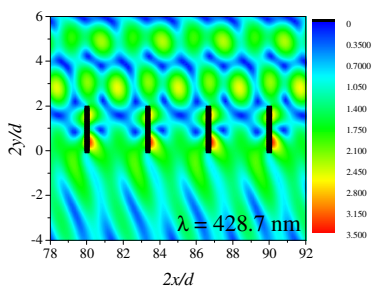

(b)

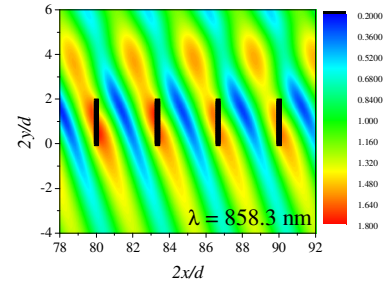

(d)
Fig. 5 In-resonance magnetic near-field patterns near four central strips for the $N=50$ strip grating with geometry of Fig.4 under the inclined incidence $\beta=$ $\pi / 4$ : cavity resonance at $\lambda=506.5 \mathrm{~nm}$ (a), surface-plasmon at $\lambda=384.2 \mathrm{~nm}$ (b), and two grating resonances at $\lambda=858.3 \mathrm{~nm}$ (c) and $\lambda=428.7 \mathrm{~nm}$ (d).

\section{REFERENCES}

[1] T. B. A. Senior and J. L. Volakis, "Sheet simulation of a thin dielectric layer," Radio Sci., vol. 22, no. 7, pp. 1261-1272, 1987.

[2] E. Bleszynski, M. Bleszynski, and T. Jaroszewicz, "Surface-integral equations for EM scattering from impenetrable and penetrable sheets," IEEE Antennas Propag. Mag., vol. 36, no 6, pp. 14-25, 1993.

[3] Z. Nazarchuk and K. Kobayashi, "Mathematical modelling of electromagnetic scattering from a thin penetrable target," Progr. Electromagn. Res., vol. 55, pp. 95-116, 2005.

[4] O. V. Shapoval, R. Sauleau, and A. I. Nosich, "Scattering and absorption of waves by flat material strips analyzed using generalized boundary conditions and Nystrom-type algorithm," IEEE Trans. Antennas and Propagation, vol. 59, no 9, pp. 3339-3346, 2011.

[5] O. V. Shapoval and A. I. Nosich, "Finite gratings of many thin silver nanostrips: optical resonances and role of periodicity," AIP Advances, vol. 3, no 4, pp. 042120/13, 2013.

[6] T. L. Zinenko, M. Marciniak, and A. I. Nosich, "Accurate analysis of light scattering and absorption by an infinite flat grating of thin silver nanostrips in free space using the method of analytical regularization," IEEE J. Selected Topics in Quantum Electronics, vol. 19, no 3, pp. 9000108/8, 2013.

[7] O. V. Shapoval, J. Ctyroky, and A. I. Nosich, "Resonance effects in the optical antennas shaped as finite comb-like gratings of noble-metal nanostrips, " Proc. SPIE, Integrated Optics: Physics and Simulation, vol. 8781, 87810U/8, 2013.

[8] I. O. Sukharevsky, O. V. Shapoval, A. Altintas, and A. I. Nosich, "Validity and limitations of the median-line integral equation technique in the scattering by material strips of sub-wavelength thickness, " IEEE Trans. Antennas and Propagation, vol. 62, 2014, will be published.

[9] J. Tsalamengas, "Exponentially converging Nystrom's methods for systems of SIEs with applications to open/closed strip or slot-loaded 2D structures," IEEE Trans. Antennas Propag., vol. 54, pp. 1549-1558, 2006.

[10] A. A. Nosich, Y. V. Gandel, A. Matsushima, and R. Sauleau, "Collimation and focusing of wave beams with metal-plate lens antennas analyzed using Nystrom-type MDS algorithm," Proc. IEEE AP-S Int. Symp. (APS-2008), San Diego, 2008, art. no 237.10.

P. B. Johnson and R. W. Christy, "Optical constants of the noble metals," Phys. Rev. B, vol. 6, pp. 4370-4379, 197 